Publ. RIMS, Kyoto Univ.

36 (2000), 85-109

\title{
The Meromorphic Solutions of the Bruschi-Calogero Equation
}

By

Nariya $\mathrm{K}_{\mathrm{AWAZUmI}}{ }^{*}$ and Youichi Shibukawa ${ }^{* *}$

\begin{abstract}
We give all the meromorphic functions defined near the origin $0 \in \mathbb{C}$ satisfying a functional equation investigated by Bruschi and Calogero [1], [2].
\end{abstract}

\section{§ 0. Introduction}

It is an important problem to find a Lax pair $\mathbf{L}$ and $\mathbf{M}$ whose equations of motion are equivalent to the Lax equation [10], [11], [12]. In order to prove their complete integrability it is convenient to use a Lax representation.

The systems of Calogero-Sutherland type, which describe one-dimensional $n$-particle dynamics, are defined by the following Hamiltonian

$$
H=\frac{1}{2} \sum_{j=1}^{n} p_{j}^{2}+U\left(q_{1}, \ldots, q_{n}\right)
$$

where the potential $U$ has the form

Communicated by T. Kawai, July 8, 1999. Revised November 1, 1999.

1991 Mathematics Subject Classification: Primary, 58F07; Secondary, 33E05, 35Q58, 39B02, 30D05.

* Department of Mathematical Sciences, University of Tokyo, Tokyo 153-8914, Japan. e-mail: kawazumi@ms.u-tokyo.ac.jp

** Department of Mathematics, Faculty of Science, Hokkaido University, Sapporo 0600810, Japan.

e-mail: shibu@math.sci.hokudai.ac.jp 


$$
U\left(q_{1}, \ldots, q_{n}\right)=g^{2} \sum_{j<k}^{n} v\left(q_{j}-q_{k}\right)
$$

Lax pairs for the system above were originated by Calogero [3] and Moser [7], and are given by the matrices

$$
\begin{aligned}
L_{j k} & =p_{j} \delta_{j k}+\sqrt{-1} g\left(1-\delta_{j k}\right) x\left(q_{j}-q_{k}\right), \\
M_{j k} & =g\left[\delta_{j k} \sum_{l \neq j} z\left(q_{j}-q_{l}\right)-\left(1-\delta_{j k}\right) y\left(q_{j}-q_{k}\right)\right] .
\end{aligned}
$$

Substituting these matrices in the Lax equation $\sqrt{-1} \stackrel{\mathbb{L}}{=}[\mathbb{M}, \mathbb{L}]$ and requiring that this equation is equivalent to the Hamiltonian equations, we get a certain functional equation for the functions $x(\xi)$ and $z(\xi)$. This functional equation has been solved in a series of papers including [4], [9]. The solutions are expressed in terms of elliptic functions, trigonometric functions or rational functions.

Later, Ruijsenaars and Schneider [15] have introduced a class of integrable dynamical systems characterized by the equations of motion

$$
\ddot{q}_{j}=\sum_{\substack{k=1 \\ k \neq j}}^{n} \dot{q}_{j} \dot{q}_{k} v\left(q_{j}-q_{k}\right), \quad q_{j}=q_{j}(t), \quad j=1,2, \ldots, n .
$$

Bruschi and Calogero [1] discovered a representation of the equations of motion of the system (0.1) in the Lax form

$$
\stackrel{\dot{L}}{=}[\mathbf{L}, \mathbf{M}] \text {, }
$$

where $\mathbb{L}$ and $\mathbb{M}$ are the $n \times n$ matrices,

$$
\begin{gathered}
L_{j k}=\delta_{j k} \dot{q}_{j}+\left(1-\delta_{j k}\right)\left(\dot{q}_{j} \dot{q}_{k}\right)^{1 / 2} \alpha\left(q_{j}-q_{k}\right), \\
M_{j k}=\delta_{j k} \sum_{\substack{m=1 \\
m \neq j}}^{n} \dot{q}_{m} \beta\left(q_{j}-q_{m}\right)+\left(1-\delta_{j k}\right)\left(\dot{q}_{j} \dot{q}_{k}\right)^{1 / 2} \gamma\left(q_{j}-q_{k}\right) .
\end{gathered}
$$

Here the function $\alpha(x)$ is a solution of the following functional equation of addition type

$$
\alpha(x) \alpha^{\prime}(y)-\alpha^{\prime}(x) \alpha(y)=(\alpha(x+y)-\alpha(x) \alpha(y))(\eta(x)-\eta(y))
$$

which we call the Bruschi-Calogero equation. The function $v(x)$ is given by 


$$
v(x)=\frac{d}{d x} \log (\alpha(x) \alpha(-x)-1) .
$$

Bruschi and Calogero [1], [2] have investigated general analytic solutions of this functional equation (0.2). They have obtained some solutions $\alpha$ expressed by elliptic functions in the most general case, and they had some trigonometric and rational solutions by degenerating the periods of the elliptic functions.

The main purpose of the present paper is to solve the functional equation (0.2) in the most rigorous way. More precisely, we shall give all meromorphic solutions of the functional equation (0.2) defined near the origin $0 \in \mathbb{C}$.

Theorem 0.1. Let $\alpha$ and $\eta$ be holomorphic functions defined on $a$ punctured disk $\{x \in \mathbb{C} ; 0<|x|<r\}$ for some $r>0$. If they satisfy the functional equation (0.2), then they are equal to one of the following functions.

(0-i)

$$
\alpha(x)=0 \text { or } e^{\rho x}, \eta \text { : arbitrary, }(\rho \in \mathbb{C})
$$

$$
\alpha(x)=C e^{\rho x}, \eta: \text { constant },(C, \rho \in \mathbb{C}, C \neq 0,1)
$$

$$
\begin{aligned}
& \alpha(x)=e^{\rho x} \frac{\sigma\left(\mu ; \tau_{1}, \tau_{2}\right) \sigma\left(\lambda x+\nu ; \tau_{1}, \tau_{2}\right)}{\sigma\left(\nu ; \tau_{1}, \tau_{2}\right) \sigma\left(\lambda x+\mu ; \tau_{1}, \tau_{2}\right)}, \\
& \eta(z)=\lambda \zeta\left(\lambda z ; \tau_{1}, \tau_{2}\right)-\lambda \zeta\left(\lambda z+\mu ; \tau_{1}, \tau_{2}\right)+A, \\
& \left(\rho, \mu, \nu, A \in \mathbb{C}, \lambda, \tau_{1}, \tau_{2} \in \mathbb{C} \backslash\{0\}, \quad \operatorname{Im} \tau_{2} / \tau_{1}>0, \mu, \nu \notin \mathbb{Z} \tau_{1}+\mathbb{Z} \tau_{2}\right)
\end{aligned}
$$

(II)

$$
\begin{aligned}
& \alpha(x)= \\
& \begin{array}{l}
\rho x \\
c\left(e^{2 x / \lambda}-1\right)+b
\end{array} \\
& \begin{aligned}
& \eta(x)=\frac{2 \lambda^{-1} e^{2 x / \lambda}}{e^{2 x / \lambda}-1}-\frac{2 \lambda^{-1} c e^{2 x / \lambda}}{c\left(e^{2 x / \lambda}-1\right)+b}+A, \\
&(\lambda, a, b, c, A \in \mathbb{C}, \lambda \neq 0, \\
&\quad b(a-c) \neq 0, a \neq 0 \text { or } b \neq c, c \neq 0 \text { or } a \neq b)
\end{aligned}
\end{aligned}
$$


(III)

$$
\begin{gathered}
\alpha(x)=e^{\rho x} \frac{a x+b}{c x+b}, \quad \eta(x)=\frac{b}{x(c x+b)}+A \\
(\rho, a, b, c, A \in \mathbb{C}, b(a-c) \neq 0)
\end{gathered}
$$

Here $\sigma\left(x ; \tau_{1}, \tau_{2}\right)$ is the Weierstrass sigma function, and $\zeta\left(x ; \tau_{1}, \tau_{2}\right)$ the Weierstrass zeta function. All the solutions except for the case (0-i) extend themselves to meromorphic functions defined on the whole plane $\mathbb{C}$.

It should be remarked that a meromorphic function defined near the origin $0 \in \mathbb{C}$ is holomorphic on a sufficiently small punctured disk. Hence our result covers all the meromorphic solutions defined near the origin $0 \in \mathbb{C}$.

The methods we use in this paper are quite different from those of Bruschi and Calogero [1], [2].

The outline to get all meromorphic solutions is as follows. First, we shall show that the solution $\eta$ is the logarithmic derivative of some meromorphic function $\varphi$, and that the set of zeroes of $\varphi$ is a discrete subgroup of $\mathbb{C}$. As is known, such a subgroup is isomorphic to $\mathbb{Z}^{2}, \mathbb{Z}$ or $\{0\}$. If this subgroup is isomorphic to $\mathbb{Z}^{2}$, we find out that $\eta$ and $\alpha$ are expressed in terms of elliptic functions by a standard argument. In other two cases, the key tool to obtaining the explicit form of the solution $\alpha$ is the great Picard theorem. (See, for example, [5].) As a result, we shall show that $\alpha$ is expressed in terms of trigonometric functions or rational functions.

After the first draft of this paper was completed, we were informed that Ochiai, Oshima and Sekiguchi [8], [13] have studied all the completely integrable systems with the invariance under the action of the Weyl groups. In their papers, they solved the functional differential equations of the potential function. We should note that these functional differential equations are of addition type also.

\section{Acknowledgement}

The authors would like to express their gratitude to Professor Toshio Oshima and the referee for several valuable comments. They owe, in particular, a suggestion about the defining domains of solutions to Professor Oshima. The first author would like to thank Professor Yukio Matsumoto for helpful discussions. 


\section{$\S 1$. Equivalence of Two Functional Equations}

In this section we prove that the equation (0.2) and the functional equation

$$
\alpha(x+y)-\alpha(x) \alpha(y)=\varphi(x) \varphi(y) \phi(x+y)
$$

for meromorphic functions $\alpha, \varphi$ and $\phi$ are equivalent to each other (Proposition 1.3). As is easily shown, if we use a coordinate system $(s, t)$ on the plane $\mathbb{C}^{2}$ given by

$$
\left\{\begin{array}{l}
s:=x+y \\
t:=x
\end{array}\right.
$$

the equation (1.1) is equivalent to

$$
\alpha(s)-\alpha(t) \alpha(s-t)=\varphi(t) \varphi(s-t) \phi(s) .
$$

Moreover we show that almost all holomorphic solutions of the equation (0.2) defined on a small punctured disk extend themselves to meromorphic functions defined near the origin $0 \in \mathbb{C}$ (Lemma 1.2).

The exponential function $\alpha(x)=C e^{\rho x}$ for any $C$ and $\rho \in \mathbb{C}$ is a solution of both of the equations (0.2) and (1.1). If $C=0$ or 1 , then one of $\varphi$ and $\phi$ is 0 , and the other one and $\eta$ may be chosen arbitrarily. On the other hand, if $C \neq 0,1$, then $\eta(x)=A$, a constant function, $\varphi(x)=C_{1} e^{A x}$ for some non-zero constant $C_{1}$, and $\phi(x)=$ $\left(C-C^{2}\right) C_{1}^{-2} e^{(\rho-A) x}$. These exponential solutions will often disturb our train of demonstrations. So, throughout this paper, we call them obvious solutions to discriminate them from other non-trivial solutions.

Lemma 1.1. Let $\alpha=\alpha(x)$ be a holomorphic function defined on a punctured disk $\{x \in \mathbb{C} ; 0<|x|<r\}$ for some $r>0$. Suppose $\alpha$ satisfies one of the equations

(1) $\alpha(x) \alpha^{\prime}(y)-\alpha^{\prime}(x) \alpha(y)=0$,

(2) $\alpha$ extends itself to a holomorphic function defined near $0 \in \mathbb{C}$, and $\alpha(0) \alpha(x+y)-\alpha(x) \alpha(y)=0$,

(3) $\alpha(x+y)-\alpha(x) \alpha(y)=0$,

where $x, y$ and $x+y$ run over the defining domain. Then there exist $C$ and $\rho \in \mathbb{C}$ such that $\alpha(x)=C e^{\rho x}$. 
Proof. When $\alpha=0$, the lemma is trivial. So we may assume $\alpha \neq 0$. The equation (3) implies (2). In fact, choose a point $x$ in the punctured disk $\{0<|x|<r\}$. Then $\lim _{y \rightarrow 0} \alpha(y)=\lim _{y \rightarrow 0} \alpha(x+y) / \alpha(x)=1$. So we have $\alpha(0)=1 \neq \infty$. Thus we obtain (2).

The equation (2) implies (1). If we substitute $x=t$ and $y=s-t$ into (2), then we have $\alpha(0) \alpha(s)-\alpha(t) \alpha(s-t)=0$. Differentiate it by the variable $t$. Then we have $\alpha(t) \alpha^{\prime}(s-t)-\alpha^{\prime}(t) \alpha(s-t)=0$, which is equivalent to (1).

From the equation (1) we have $\alpha^{\prime}(y) / \alpha(y)=\alpha^{\prime}(x) / \alpha(x)$ for any $x$ and $y$ in the defining domain. In other words, $\alpha^{\prime} / \alpha$ is a constant function. Hence there exist $C$ and $\rho \in \mathbb{C}$ such that $\alpha(x)=C e^{\rho x}$.

Next we prove that the solutions have no essential singularities at 0 $\in \mathbb{C}$

Lemma 1.2. Let $\alpha=\alpha(x)$ and $\eta=\eta(x)$ be holomorphic functions defined on a punctured disk $\{x \in \mathbb{C} ; 0<|x|<r\}$ for some $r>0$. Suppose the pair $(\alpha, \eta)$ is a non-obvious solution of the functional equation (0.2). Then the functions $\alpha$ and $\eta$ extend themselves to meromorphic functions defined near the origin $0 \in \mathbb{C}$.

Proof. Since $(\alpha, \eta)$ is not obvious, we have $\eta(x)-\eta(y) \neq 0$, and $\alpha(x+y)-\alpha(x) \alpha(y) \neq 0$, as functions in $(x, y)$. (See Lemma 1.1.) Fix an arbitrary point $t_{0}$ in the punctured disk. Then (0.2) implies

$$
\alpha(s)=\alpha\left(t_{0}\right) \alpha\left(s-t_{0}\right)+\frac{\alpha\left(t_{0}\right) \alpha^{\prime}\left(s-t_{0}\right)-\alpha^{\prime}\left(t_{0}\right) \alpha\left(s-t_{0}\right)}{\eta\left(t_{0}\right)-\eta\left(s-t_{0}\right)}
$$

for any $s, 0<|s|<r-\left|t_{0}\right|$. The right-hand side extends itself to a meromorphic function near $s=0$. Hence $\alpha(s)$ is meromorphic at $s=0$.

Fix a point $y_{0}$ in the punctured disk. From (0.2) again, we have

$$
\eta(x)=\eta\left(y_{0}\right)+\frac{\alpha(x) \alpha^{\prime}\left(y_{0}\right)-\alpha^{\prime}(x) \alpha\left(y_{0}\right)}{\alpha\left(x+y_{0}\right)-\alpha(x) \alpha\left(y_{0}\right)}
$$

for any $x, 0<|x|<r-\left|y_{0}\right|$. The right-hand side is meromorphic at $x=$ 0 , and so is the function $\eta(x)$.

Thus, in what follows, we may assume that the solutions are meromorphic functions defined near the origin $0 \in \mathbb{C}$.

Now we can prove the equivalence of the two equations (0.2) and (1.1).

Proposition 1.3. The two functional equations (0.2) and (1.1) for 
meromorphic functions defined on the whole plane $\mathbb{C}$ (or defined near the origin $0 \in \mathbb{C}$ ) are equivalent to each other. More precisely, we have

(1) If $(\alpha, \varphi, \phi)$ is a non-obvious solution of the equation (1.1), the pair $(\alpha, \eta)$ given by $\eta(x)=\varphi^{\prime}(x) / \varphi(x)$ satisfies the equation $(0.2)$.

(2) If two non-obvious solutions $\left(\alpha_{1}, \varphi_{1}, \phi_{1}\right)$ and $\left(\alpha_{2}, \varphi_{2}, \phi_{2}\right)$ of the equation (1.1) satisfy $\alpha_{1}=\alpha_{2}$ and $\varphi_{1}^{\prime}(x) / \varphi_{1}(x)=\varphi_{2}^{\prime}(x) / \varphi_{2}(x)$, then we have $\varphi_{2}=C^{\prime} \varphi_{1}$ and $\phi_{2}=C^{\prime-2} \phi_{1}$ for some non-zero constant $C^{\prime} \in \mathbb{C}$.

(3) For any non-obvious solution $(\alpha, \eta)$ of the equation $(0.2)$, there exist meromorphic functions $\varphi$ and $\phi$, such that $\eta(x)=\varphi^{\prime}(x) / \varphi(x)$ and the triple $(\alpha, \varphi, \phi)$ satisfies the equation (1.1).

In other words, if the multiplicative group $\mathbb{C}-\{0\}$ acts on the set of non-obvious solutions of the equation (1.1) by $C^{\prime}(\alpha, \varphi, \phi):=$ $\left(\alpha, C^{\prime} \varphi, C^{\prime-2} \psi\right), C^{\prime} \in \mathbb{C}-\{0\}$, then the orbit space is naturally isomorphic to the set of all non-obvious solutions of the equation (0.2).

Proof. In view of Lemma 1.1, for any non-obvious solution $\alpha$, we have $\alpha(x+y)-\alpha(x) \alpha(y) \neq 0$ and $\alpha(x) \alpha^{\prime}(y)-\alpha^{\prime}(x) \alpha(y) \neq 0$. Hence, if $(\alpha, \varphi, \phi)$ is a non-obvious solution of (1.1), then we have $\varphi \neq 0$ and $\phi \neq$ 0 . Especially the logarithmic derivative $\varphi^{\prime} / \varphi$ is a well-defined meromorphic function.

(1) Differentiating the equation (1.3) by the variable $t$, we obtain (1) immediately.

(2) Suppose $\varphi_{1}^{\prime}(x) / \varphi_{1}(x)=\varphi_{2}^{\prime}(x) / \varphi_{2}(x)$. Then $\frac{d}{d x} \log \left(\varphi_{2}(x) / \varphi_{1}(x)\right)$ $=0$, which proves $(2)$.

(3) Let $(\alpha, \eta)$ be a non-obvious solution of (0.2). Using the coordinate system (1.2), we obtain

$$
\frac{\partial}{\partial t} \log (\alpha(s)-\alpha(t) \alpha(s-t))=\eta(t)-\eta(s-t) .
$$

Lemma 1.4. $\operatorname{ord}_{p} \eta=-1$ and $\operatorname{Res}_{p} \eta \in \mathbb{Z}$ for any pole $p \in \mathbb{C}$ of the function $\eta$.

Proof of Lemma 1.4. Let $\lambda \in \mathbb{C}$ be a pole of the function $\eta$. Choose a generic $s_{0} \in \mathbb{C}$. Then the singular part of $\eta(t)-\eta\left(s_{0}-t\right)$ at $t=\lambda$ coincides with that of $\eta(t)$. On the other hand, the equation (1.4) implies that the function $\eta(t)-\eta\left(s_{0}-t\right)$ is equal to the logarithmic derivative of the function $\alpha\left(s_{0}\right)-\alpha(t) \alpha\left(s_{0}-t\right)$. Thus the order of $\eta(t)$ at the point $\lambda$ is -1 , and its residue is equal to the order of $\alpha\left(s_{0}\right)-\alpha(t) \alpha\left(s_{0}-t\right)$ at the point $t=\lambda$. This means the residue is an integer, as was to be shown.

Now suppose $\alpha$ and $\eta$ are meromorphic functions defined on the 
whole plane $\mathbb{C}$. Then Lemma 1.4 means that the differential equation

$$
\eta(x)=\varphi^{\prime}(x) / \varphi(x)
$$

has a local meromorphic solution $\varphi$ near any point of the whole plane $\mathbb{C}$. Since the plane $\mathbb{C}$ is simply connected, such local meromorphic solutions extend themselves to the global solution $\varphi$ defined on the whole $\mathbb{C}$. Then the equation (1.4) implies

$$
\frac{\partial}{\partial t} \log \left(\frac{\alpha(s)-\alpha(t) \alpha(s-t)}{\varphi(t) \varphi(s-t)}\right)=0,
$$

so that there exists a meromorphic function $\psi(s)$ defined on the whole plane $\mathbb{C}$ such that $\alpha(s)-\alpha(t) \alpha(s-t)=\varphi(t) \varphi(s-t) \psi(s)$ for any $s$ and $t$ $\in \mathbb{C}$. Substituting (1.2) into this equation, we find out $(\alpha, \varphi, \phi)$ is a non-obvious solution of (1.1). As for local solutions, the situation is simpler.

This completes the proof of Proposition 1.3.

Remark 1.5. As is easily deduced from (1.4), if two non-obvious solutions $\left(\alpha_{1}, \eta_{1}\right)$ and $\left(\alpha_{2}, \eta_{2}\right)$ of the equation (0.2) satisfy $\alpha_{1}=\alpha_{2}$, then the difference $\eta_{2}-\eta_{1}$ is a constant function. This, together with Proposition 1.3, enables us to consider a single function $\alpha$ as a non-obvious solution of the equations (0.2) and (1.1) instead of a pair $(\alpha, \eta)$ and a triple $(\alpha, \varphi, \psi)$.

\section{§ 2. Behavior of Solutions Near the Origin}

In what follows, we confine ourselves to non-obvious meromorphic solutions $\alpha, \eta, \varphi$ and $\phi$ of the functional equations (0.2) and (1.1). We remark $\eta(x)-\eta(y) \neq 0$ and $\varphi \neq 0$, since $\alpha$ is not obvious. We consider local solutions, i.e., solutions defined near the origin, and global solutions, i.e., solutions defined on the whole plane $\mathbb{C}$, simultaneously.

\section{Lemma 2.1.}

(1) Let $p \in \mathbb{C}$ be a point in the defining domain of solutions $\alpha, \eta, \varphi$ and $\psi$. Then $p$ is a pole of the function $\varphi$, if and only if $p$ is a pole of the function $\alpha$.

(2) If $p \in \mathbb{C}$ is a pole of $\varphi$, then the orders of $\alpha$ and $\varphi$ at $p$ are equal to each other, i.e., $\operatorname{ord}_{p} \varphi=\operatorname{ord}_{p} \alpha$. Especially we have $(\alpha / \varphi)(p) \neq 0, \infty$.

(3) A point $p \in \mathbb{C}$ is a zero of the function $\varphi$, if and only if the equation $\alpha(s)=\alpha(p) \alpha(s-p)$ holds for all $s$ in the defining domain of $\alpha$. 
Proof. Choose a generic $s_{0} \in \mathbb{C}$ such that all of $\alpha\left(s_{0}-p\right), \alpha\left(s_{0}\right)$, $\varphi\left(s_{0}-p\right)$ and $\phi\left(s_{0}\right)$ are neither 0 nor $\infty$. Then we have

$$
\operatorname{ord}_{p}\left(\alpha\left(s_{0}\right)-\alpha(t) \alpha\left(s_{0}-t\right)\right)=\operatorname{ord}_{p}\left(\varphi(t) \varphi\left(s_{0}-t\right)\right)=\operatorname{ord}_{p} \varphi,
$$

since $\left(\alpha\left(s_{0}\right)-\alpha(t) \alpha\left(s_{0}-t\right)\right) \varphi(t)^{-1} \varphi\left(s_{0}-t\right)^{-1}=\psi\left(s_{0}\right) \neq 0, \infty$, and $\varphi\left(s_{0}-p\right)$ $\neq 0, \infty$.

Now suppose $\varphi(p)=\infty$. Then $\alpha\left(s_{0}\right)-\alpha(p) \alpha\left(s_{0}-p\right)=\infty$. We have $\alpha(p)=\infty$, since $\alpha\left(s_{0}-p\right), \alpha\left(s_{0}\right) \neq 0, \infty$. Moreover $\operatorname{ord}_{p}(\varphi)=$ $\operatorname{ord}_{p}\left(\alpha\left(s_{0}\right)-\alpha(t) \alpha\left(s_{0}-t\right)\right)=\operatorname{ord}_{p}\left(\alpha(t) \alpha\left(s_{0}-t\right)\right)=\operatorname{ord}_{p} \alpha . \quad$ Conversely suppose $\alpha(p)=\infty$. Then $\alpha\left(s_{0}\right)-\alpha(p) \alpha\left(s_{0}-p\right)=\infty$, so that $\varphi(p)=\infty$ by (2.1).

Finally suppose $\varphi(p)=0$. Then (2.1) implies $\alpha\left(s_{0}\right)-\alpha(p) \alpha\left(s_{0}-p\right)=$ 0 for generic $s_{0}$. Hence $\alpha(s)=\alpha(p) \alpha(s-p)$ for all $s$ in the defining domain of $\alpha$. Conversely we assume $\alpha(s)=\alpha(p) \alpha(s-p)$ for all $s$ in the defining domain. Then (2.1) implies $\varphi(p)=0$.

This completes the proof of Lemma 2.1.

Our main purpose in this section is to prove the following.

Lemma 2.2. Let $\alpha, \eta, \varphi$ and $\phi$ be non-obvious meromorphic solutions of (0.2) and (1.1) defined near the origin $0 \in \mathbb{C}$. Then we have $\varphi(0)=0$, $\alpha(0)=1, \varphi^{\prime}(0) \neq 0$ and

$$
\psi(s)=\left(\frac{\alpha^{\prime}(s)}{\alpha(s)}-\frac{\alpha^{\prime}(0)}{\alpha(0)}\right) \frac{\alpha(s)}{\varphi^{\prime}(0) \varphi(s)} .
$$

Proof. We begin by proving that $\varphi$ and $\alpha$ are holomorphic near the origin $0 \in \mathbb{C}$.

Assume $\varphi(0)=\infty$. Then $\alpha(0)=\infty$ and $(\alpha / \varphi)(0) \neq 0, \infty$ from Lemma 2.1. The equation (1.3) implies

$$
\varphi(t)^{-1} \alpha(s) \varphi(s-t)^{-1}-(\alpha / \varphi)(t)(\alpha / \varphi)(s-t)=\phi(s) .
$$

We obtain $\psi(s)=-(\alpha / \varphi)(0)(\alpha / \varphi)(s)$ as $t \rightarrow 0$. Hence we have

$$
-(\alpha / \varphi)(0)(\alpha / \varphi)(s) \frac{1}{\alpha(s)}=\frac{\phi(s)}{\alpha(s)}=\varphi(t)^{-1} \varphi(s-t)^{-1}\left(1-\frac{\alpha(t) \alpha(s-t)}{\alpha(s)}\right)
$$

so that $\varphi(t)^{-1} \varphi(-t)^{-1}=0$ as $s \rightarrow 0$. This means $\varphi^{-1}=0$, and so contradicts the fact $\varphi$ is holomorphic on a sufficiently small punctured disk centered at the origin. Therefore we find out $\varphi(0) \neq \infty$. From Lemma 2.1 we have $\alpha(0) \neq \infty$. 
Thus $\varphi$ and $\alpha$ are holomorphic near the origin. Especially we may substitute $x=0$ into the derivatives $\alpha^{\prime}(x)$ and $\varphi^{\prime}(x)$. Differentiate the equation (1.3) by the variable $t$, and substitute $t=0$ into it. Then we obtain

$$
\alpha(0) \alpha^{\prime}(s)-\alpha^{\prime}(0) \alpha(s)=\phi(s)\left(\varphi^{\prime}(0) \varphi(s)-\varphi(0) \varphi^{\prime}(s)\right) .
$$

Next, in order to prove $\varphi(0)=0$, assume $\varphi(0) \neq 0$. Substituting $t=$ 0 into the equation (1.3), we obtain

$$
\psi(s)=\frac{(1-\alpha(0)) \alpha(s)}{\varphi(0) \varphi(s)} .
$$

This, together with the equation (2.3), implies

$$
\alpha(0) \frac{\alpha^{\prime}(s)}{\alpha(s)}-\alpha^{\prime}(0)=(1-\alpha(0))\left(\frac{\varphi^{\prime}(0)}{\varphi(0)}-\frac{\varphi^{\prime}(s)}{\varphi(s)}\right) .
$$

Hence we have

$$
\begin{aligned}
\alpha(0) \frac{\alpha^{\prime}(x)}{\alpha(x)}+(1-\alpha(0)) \frac{\varphi^{\prime}(x)}{\varphi(x)} & =\alpha^{\prime}(0)+(1-\alpha(0)) \frac{\varphi^{\prime}(0)}{\varphi(0)} \\
& =\alpha(0) \frac{\alpha^{\prime}(y)}{\alpha(y)}+(1-\alpha(0)) \frac{\varphi^{\prime}(y)}{\varphi(y)}
\end{aligned}
$$

and so

$$
\begin{aligned}
(1-\alpha(0))(\eta(x)-\eta(y)) & =(1-\alpha(0))\left(\frac{\varphi^{\prime}(x)}{\varphi(x)}-\frac{\varphi^{\prime}(y)}{\varphi(y)}\right) \\
& =\alpha(0)\left(\frac{\alpha^{\prime}(y)}{\alpha(y)}-\frac{\alpha^{\prime}(x)}{\alpha(x)}\right) \\
& =\alpha(0)\left(\frac{\alpha(x+y)}{\alpha(x) \alpha(y)}-1\right)(\eta(x)-\eta(y)) .
\end{aligned}
$$

Here recall $\eta(x)-\eta(y) \neq 0$, since $\alpha$ is not obvious. Therefore we have $\alpha(0) \alpha(x+y)=\alpha(x) \alpha(y)$. This means $\alpha$ is obvious from Lemma 1.1, and contradicts our assumption.

Therefore we obtain $\varphi(0)=0$. From Lemma 2.1 (3) we have $\alpha(s)-\alpha(0) \alpha(s)=0$. Hence $\alpha(0)=1$. Now the formula (2.3) turns out to be

$$
\alpha^{\prime}(s)-\alpha^{\prime}(0) \alpha(s)=\phi(s) \varphi^{\prime}(0) \varphi(s)
$$


Since $\alpha$ is not obvious, we have $\varphi^{\prime}(0) \neq 0$. Hence we have $\phi(s)=$ $\left(\alpha^{\prime}(s) / \alpha(s)-\alpha^{\prime}(0) / \alpha(0)\right) \alpha(s) / \varphi^{\prime}(0) \varphi(s)$, as was to be shown.

The formula (2.2) means

$$
\frac{\alpha(s)-\alpha(t) \alpha(s-t)}{\varphi(t) \varphi(s-t)}=\frac{\alpha^{\prime}(s)-\alpha^{\prime}(0) \alpha(s)}{\varphi^{\prime}(0) \varphi(s)},
$$

which plays an important role throughout this paper.

As a consequence of the relation (2.2), we obtain

Proposition 2.3. Any non-obvious meromorphic solutions of the equations (0.2) and (1.1) defined near the origin extend themselves to those defined on the whole plane $\mathbb{C}$.

Proof. Let $\alpha, \eta, \varphi$ and $\phi$ be non-obvious meromorphic solutions of the equations (0.2) and (1.1) defined on a disk $\{x \in \mathbb{C} ;|x|<r\}$ for some $r>0$. From (0.2) we have

$$
\alpha(x+y)=\alpha(x) \alpha(y)+\frac{\alpha(x) \alpha^{\prime}(y)-\alpha^{\prime}(x) \alpha(y)}{\eta(x)-\eta(y)},
$$

which implies $\alpha$ extends itself to $\{x \in \mathbb{C} ;|x|<2 r\}$. From the equation (1.1)

$$
\phi(x+y)=\frac{\alpha(x+y)-\alpha(x) \alpha(y)}{\varphi(x) \varphi(y)}
$$

and the equation (2.2)

$$
\varphi(s)=\left(\frac{\alpha^{\prime}(s)}{\alpha(s)}-\frac{\alpha^{\prime}(0)}{\alpha(0)}\right) \frac{\alpha(s)}{\varphi^{\prime}(0) \phi(s)},
$$

$\phi$ and $\varphi$ extend themselves to $\{x \in \mathbb{C} ;|x|<2 r\}$. Recall $\eta$ is equal to $\varphi^{\prime} / \varphi$. Such extensions satisfy the functional equations by means of the permanence of functional relations.

Consequently these solutions extend themselves to $\{x \in \mathbb{C} ;|x|<$ $2^{n} r$ for all $n \geq 1$, and so to the whole plane $\mathbb{C}$. This completes the proof.

\section{§ 3. Discrete Subgroups}

In view of Proposition 2.3 we may confine ourselves to non-obvious meromorphic solutions defined on the whole plane $\mathbb{C}$.

We denote the zeroes of $\varphi$, the poles of $\varphi$ and the zeroes of $\alpha$ by $\Lambda_{+}$, 
$\Lambda_{-}$and $\Lambda^{\alpha}$, respectively. Clearly these subsets $\Lambda_{+}, \Lambda_{-}$and $\Lambda^{\alpha}$ are all discrete subsets in the plane $\mathbb{C}$. In this section we study these discrete subsets.

By Lemma 2.1 (3) we have

$$
\Lambda_{+}=\{u \in \mathbb{C} ; \alpha(t+u)=\alpha(u) \alpha(t), \quad \forall t \in \mathbb{C}\} .
$$

Since $\varphi(0)=0$, we have $\Lambda_{+} \neq \emptyset$. One can deduce easily the following lemma from the identification (3.1).

Lemma 3.1. The discrete subset $\Lambda_{+}$is a subgroup of the additive group $\mathbb{C}$. Moreover the restriction of $\alpha$ to $\Lambda_{+}$gives a homomorphism of $\Lambda_{+}$ into the multiplicative group $\mathbb{C} \backslash\{0\}$. Especially $\alpha(u) \neq 0$ for any $u \in \Lambda_{+}$.

As is known, any discrete subgroup $\Lambda_{+}$of the additive group $\mathbb{C}$ is given by one of the following.

(1) There exist $\tau_{1}$ and $\tau_{2} \in \mathbb{C} \backslash\{0\}$ such that $\operatorname{Im} \tau_{2} / \tau_{1}>0$ and $\Lambda_{+}=$ $\mathbb{Z} \tau_{1}+\mathbb{Z} \tau_{2}$. In what follows, we call such a case non-degenerate.

(2) There exists $\lambda_{0} \in \mathbb{C} \backslash\{0\}$ such that $\Lambda_{+}=\mathbb{Z} \lambda_{0}$. We will consider this case in $\S 6$.

(3) $\Lambda_{+}=\{0\}$. We will consider this case in $\S 5$.

(See, for example, Pontryagin [14] ch. 3, § 19, example 33.) In short, we obtain

$$
\Lambda_{+} \cong \mathbb{Z}^{2}, \mathbb{Z} \text { or }\{0\} .
$$

In the succeeding sections, in the case where this subgroup is isomorphic to $\mathbb{Z}^{2}$, we prove that $\alpha$ and $\eta$ are expressed in terms of elliptic functions by a standard argument. In other two cases, we shall show that $\alpha$ and $\eta$ are expressed in terms of trigonometric functions or rational functions.

Next we study the discrete subsets $\Lambda_{-}$and $\Lambda^{\alpha}$.

Lemma 3.2. If $p \in \Lambda_{-}$, namely, $p$ is a pole of $\varphi$ and $\alpha$, then

$$
\varphi(p-t) \varphi(t)=-\varphi^{\prime}(0) \operatorname{Res}_{p} \varphi \neq 0,
$$

and $\operatorname{ord}_{p} \varphi=\operatorname{ord}_{p} \alpha=-1$.

Proof. Choose a generic $t_{0} \in \mathbb{C}$ such that all of $\varphi\left(t_{0}\right), \varphi\left(p-t_{0}\right)$, $\alpha\left(t_{0}\right)$ and $\alpha\left(p-t_{0}\right)$ are neither 0 nor $\infty$. From the equation (2.4) we have 


$$
\frac{\varphi^{\prime}(0)}{\varphi\left(t_{0}\right) \varphi\left(s-t_{0}\right)}\left(1-\frac{\alpha\left(t_{0}\right) \alpha\left(s-t_{0}\right)}{\alpha(s)}\right)=\frac{1}{\varphi(s)}\left(\frac{\alpha^{\prime}(s)}{\alpha(s)}-\alpha^{\prime}(0)\right) .
$$

The left-hand side turns out to be $\varphi^{\prime}(0) /\left(\varphi\left(t_{0}\right) \varphi\left(p-t_{0}\right)\right) \neq 0, \infty$ as $s \rightarrow p$. Hence

$$
\lim _{s \rightarrow p} \frac{1}{\varphi(s)}\left(\frac{\alpha^{\prime}(s)}{\alpha(s)}-\alpha^{\prime}(0)\right) \neq 0, \infty,
$$

so that $\operatorname{ord}_{p} \varphi=\operatorname{ord}_{p}\left(\alpha^{\prime}(s) / \alpha(s)-\alpha^{\prime}(0)\right)$. Since $\operatorname{ord}_{p} \varphi<0, s=p$ is a pole of $\alpha^{\prime}(s) / \alpha(s)-\alpha^{\prime}(0)$. Hence we have $\operatorname{ord}_{p}\left(\alpha^{\prime}(s) / \alpha(s)-\alpha^{\prime}(0)\right)=$ $\operatorname{ord}_{p}\left(\alpha^{\prime}(s) / \alpha(s)\right)=-1$. Thus we obtain $\operatorname{ord}_{p} \alpha=\operatorname{ord}_{p} \varphi=-1$. (See Lemma $2.1(2)$.)

Now $\varphi(s)=(s-p)^{-1} f(s)$ and $\alpha^{\prime}(s) \alpha(s)^{-1}-\alpha^{\prime}(0)=(s-p)^{-1} \beta(s)$ for some holomorphic functions $f$ and $\beta$ defined near $p$. Then $\beta(p)=$ $\operatorname{Res}_{p}\left(\alpha^{\prime} / \alpha\right)=-1, f(p)=\operatorname{Res}_{p} \varphi$, and so

$$
\lim _{s \rightarrow p} \frac{1}{\varphi(s)}\left(\frac{\alpha^{\prime}(s)}{\alpha(s)}-\alpha^{\prime}(0)\right)=\lim _{s \rightarrow p} \frac{\beta(s)}{f(s)}=\frac{-1}{\operatorname{Res}_{p} \varphi} .
$$

This means $\varphi^{\prime}(0) /\left(\varphi\left(t_{0}\right) \varphi\left(p-t_{0}\right)\right)=-1 / \operatorname{Res}_{p} \varphi$ for any generic $t_{0}$. Therefore we have

$$
\varphi(t) \varphi(p-t)=-\varphi^{\prime}(0) \operatorname{Res}_{p} \varphi
$$

for any $t \in \mathbb{C}$. Since $\alpha$ is not obvious, we have $\varphi \neq 0$ and so $-\varphi^{\prime}(0) \operatorname{Res}_{p} \varphi$ $\neq 0$. This completes the proof.

Corollary 3.3. $\Lambda_{-}$is invariant under the translation by $\Lambda_{+}$and $\#\left(\Lambda_{-} / \Lambda_{+}\right) \leq 1$.

Proof. Let $p \in \Lambda_{-}$and $u \in \Lambda_{+}$be given. Recall $\alpha(u) \neq 0, \infty$. Then we have $\alpha(p+u)=\alpha(p) \alpha(u)=\infty$, so that $p+u \in \Lambda_{-}$. This means $\Lambda_{-}$is a $\Lambda_{+}$-invariant subset.

Now, for any $p_{1}$ and $p_{2} \in \Lambda_{-}, \lim _{t \rightarrow p_{2}} \varphi\left(p_{1}-t\right) \varphi(t)=-\varphi^{\prime}(0) \operatorname{Res}_{p_{1}} \varphi$ $\neq 0, \infty$. By means of $\lim _{t \rightarrow p_{2}} \varphi(t)=\infty$, we have $\lim _{t \rightarrow p_{2}} \varphi\left(p_{1}-t\right)=0$, and so $p_{1}-p_{2} \in \Lambda_{+}$. This means $\#\left(\Lambda_{-} / \Lambda_{+}\right) \leq 1$, as was to be shown.

Lemma 3.4. Let $q \in \mathbb{C}$ be a zero of $\alpha$, i.e., $q \in \Lambda^{\alpha}$. Then we have

(1) $\alpha^{\prime}(q) \neq 0$ i.e., $\quad \operatorname{ord}_{q} \alpha=1$. 
(2) $\frac{\alpha(t) \alpha(q-t)}{\varphi(t) \varphi(q-t)}=-\frac{\alpha^{\prime}(q)}{\varphi^{\prime}(0) \varphi(q)} \neq 0$.

Moreover $\Lambda^{\alpha}$ is invariant under the translation by $\Lambda_{+}$and $\left(\Lambda^{\alpha} / \Lambda_{+}\right) \leq 1$.

Proof. Since $\Lambda_{+} \cap \Lambda^{\alpha}=\emptyset$, we have $\varphi(q) \neq 0$. From the equation (2.4) we have

$$
\frac{-\alpha(t) \alpha(q-t)}{\varphi(t) \varphi(q-t)}=\frac{\alpha^{\prime}(q)}{\varphi^{\prime}(0) \varphi(q)} .
$$

Since $\alpha / \varphi \neq 0$, we obtain (2) and $\alpha^{\prime}(q) \neq 0$, so that $\operatorname{ord}_{q} \alpha=1$.

Now let $q \in \Lambda^{\alpha}$ and $u \in \Lambda_{+}$be given. Then we have $\alpha(q+u)=$ $\alpha(q) \alpha(u)=0$, so that $q+u \in \Lambda^{\alpha}$. This means $\Lambda^{\alpha}$ is a $\Lambda_{+}$-invariant subset.

Finally, for any $q_{1}$ and $q_{2} \in \Lambda^{\alpha}$,

$$
\frac{-\alpha\left(q_{2}\right) \alpha\left(q_{1}-q_{2}\right)}{\varphi\left(q_{2}\right) \varphi\left(q_{1}-q_{2}\right)}=\frac{\alpha^{\prime}\left(q_{1}\right)}{\varphi^{\prime}(0) \varphi\left(q_{1}\right)} \neq 0, \infty .
$$

Since $\alpha\left(q_{2}\right) / \varphi\left(q_{2}\right)=0$, we have $(\alpha / \varphi)\left(q_{1}-q_{2}\right)=\infty$. It follows from Lemma 2.1 (2) that $q_{1}-q_{2} \notin \Lambda_{-}$. Thus we obtain $\varphi\left(q_{1}-q_{2}\right)=0$, i.e., $q_{1}-q_{2} \in \Lambda_{+}$. This means $\left(\Lambda^{\alpha} / \Lambda_{+}\right) \leq 1$. This completes the proof of Lemma 3.4.

\section{§4. Non-Degenerate Case}

In this section we consider the case $\Lambda_{+} \cong \mathbb{Z}^{2}$ i.e. the doubly-periodic case. The function $\alpha^{\prime} / \alpha$ admits periodicity with respect to the discrete subgroup $\Lambda_{+}$.

\section{Lemma 4.1.}

(1) The set of poles of the function $\alpha^{\prime} / \alpha$ is $\Lambda^{\alpha} \cup \Lambda_{-}$. All poles of $\alpha^{\prime} / \alpha$ are simple, and $\operatorname{Res}_{p} \alpha^{\prime} / \alpha=\operatorname{ord}_{p} \alpha$ for $p \in \Lambda^{\alpha} \cup \Lambda_{\text {.. }}$.

(2) $\left(\alpha^{\prime} / \alpha\right)(z+p)=\left(\alpha^{\prime} / \alpha\right)(z), \quad p \in \Lambda_{+}, z \in \mathbb{C}$.

(3) $\operatorname{Res}_{p} \alpha^{\prime} / \alpha=1, \quad p \in \Lambda^{\alpha}$.

(4) $\operatorname{Res}_{p} \alpha^{\prime} / \alpha=-1, \quad p \in \Lambda_{-}$.

Proof.

(1) We note that $\Lambda_{-}$is the set of the poles of $\alpha$ by Lemma 2.1 (1). By means of this, we obtain the result easily.

(2) By (3.1), we get 


$$
\alpha(t+u)=\alpha(u) \alpha(t) \quad\left(\forall t \in \mathbb{C}, \forall u \in \Lambda_{+}\right) .
$$

Then $\alpha^{\prime}(t+u)=\alpha(u) \alpha^{\prime}(t)$. From the two equations above,

$$
\frac{\alpha^{\prime}(t+u)}{\alpha(t+u)}=\frac{\alpha^{\prime}(t)}{\alpha(t)} \quad\left(\forall t \in \mathbb{C}, \forall u \in \Lambda_{+}\right) .
$$

(3) By Lemma 3.4 (1), if $p \in \Lambda^{\alpha}$, then $\operatorname{ord}_{p} \alpha=1$. Hence $\operatorname{Res}_{p}\left(\alpha^{\prime} / \alpha\right)$ $=1$ for $p \in \Lambda^{\alpha}$.

(4) From Lemma 3.2, we have $\operatorname{ord}_{p} \alpha=\operatorname{ord}_{p} \varphi=-1$ for $p \in \Lambda_{-}$. Thus $\operatorname{Res}_{p}\left(\alpha^{\prime} / \alpha\right)=-1$ for $p \in \Lambda_{-}$.

From $\Lambda_{+} \cong \mathbb{Z}^{2}$, there exist $\tau_{1}, \tau_{2}$ and $\lambda \in \mathbb{C} \backslash\{0\}$ such that Im $\tau_{2} / \tau_{1}>$ 0 and $\Lambda_{+}=\mathbb{Z}\left(\tau_{1} / \lambda\right)+\mathbb{Z}\left(\tau_{2} / \lambda\right)$.

Lemma 4.2. There exist $\mu$ and $\nu \in \mathbb{C}$ such that $-\mu / \lambda,-\nu / \lambda \notin$ $\Lambda_{+}, \Lambda_{-}=(-\mu / \lambda)+\Lambda_{+}$, and $\Lambda^{\alpha}=(-\nu / \lambda)+\Lambda_{+}$.

Proof. Suppose $\Lambda_{-}=\emptyset$ and $\Lambda^{\alpha}=\emptyset$. Then the elliptic function $\alpha^{\prime} / \alpha$ has no poles. Such an elliptic function is merely a constant, which contradicts that $\alpha$ is non-obvious.

Next we suppose that $\Lambda_{-}=\emptyset$ and $\Lambda^{\alpha} \neq \emptyset$ or that $\Lambda_{-} \neq \emptyset$ and $\Lambda^{\alpha}=\emptyset$. From Lemma 4.1 (1), the set of the poles of $\alpha^{\prime} / \alpha$ is $\Lambda^{\alpha}$ or $\Lambda_{-}$, respectively. By virtue of Corollary 3.3 and Lemma 3.4 (2), in a fundamental period-parallelogram for the elliptic function $\alpha^{\prime} / \alpha$, the number of poles of the function $\alpha^{\prime} / \alpha$ is one and the pole is simple. There is no such an elliptic function [16 p.432], [6 p.157].

Hence there exist $\mu$ and $\nu \in \mathbb{C}$ such that $-\mu / \lambda \in \Lambda_{-}$and $-\nu / \lambda \in$ $\Lambda^{\alpha}$. Because $\Lambda_{-}$and $\Lambda^{\alpha}$ are invariant under the translation by $\Lambda_{+}$, \# $\left(\Lambda_{-} / \Lambda_{+}\right) \leq 1$, and $\left(\Lambda^{\alpha} / \Lambda_{+}\right) \leq 1$, we obtain $\Lambda_{-}=(-\mu / \lambda)+\Lambda_{+}$and $\Lambda^{\alpha}$ $=(-\nu / \lambda)+\Lambda_{+}$.

We note that $\Lambda_{-} \neq \Lambda_{+}$because of the definition of $\Lambda_{-}$and $\Lambda_{+}$, and that $\Lambda^{\alpha} \neq \Lambda_{+}$by Lemma 3.1. Hence $-\mu / \lambda,-\nu / \lambda \notin \Lambda_{+}$.

We can summarize the conclusion of the function $\left(\alpha^{\prime} / \alpha\right)(z)$ just obtained as follows: $\tau_{2} / \lambda$

(1) The function $\left(\alpha^{\prime} / \alpha\right)(z)$ is doubly-periodic with periods $\tau_{1} / \lambda$,

(2) The set of poles of $\alpha^{\prime} / \alpha$ is $\left((-\nu / \lambda)+\Lambda_{+}\right) \cup\left((-\mu / \lambda)+\Lambda_{+}\right)$, and all poles are simple.

(3) $\operatorname{Res}_{p} \alpha^{\prime} / \alpha= \begin{cases}1, & p \in(-\nu / \lambda)+\Lambda_{+}, \\ -1, & p \in(-\mu / \lambda)+\Lambda_{+} .\end{cases}$ 
The Weierstrass sigma function $\sigma(z)=\rho\left(z ; \tau_{1}, \tau_{2}\right)$ is defined by

$$
\sigma\left(z ; \tau_{1}, \tau_{2}\right)=z \prod_{\substack{\omega=m_{1} \tau_{1}+m_{2} \tau_{2} \\\left(m_{1}, m_{2}\right) \in \mathbb{Z}^{2} \backslash\{(0,0)\}}}\left\{\left(1-\frac{z}{\omega}\right) \exp \left(\frac{z}{\omega}+\frac{1}{2}\left(\frac{z}{\omega}\right)^{2}\right)\right\} .
$$

Theorem 4.3.

$$
\alpha(z)=\exp (\rho z) \frac{\sigma\left(\mu ; \tau_{1}, \tau_{2}\right) \sigma\left(\lambda z+\nu ; \tau_{1}, \tau_{2}\right)}{\sigma\left(\nu ; \tau_{1}, \tau_{2}\right) \sigma\left(\lambda z+\mu ; \tau_{1}, \tau_{2}\right)}
$$

for some $\rho \in \mathbb{C}$.

Proof. By [16 p.449], [6 p.177],

$$
\frac{\alpha^{\prime}(z)}{\alpha(z)}=\rho+\zeta\left(z+\frac{\nu}{\lambda} ; \frac{\tau_{1}}{\lambda}, \frac{\tau_{2}}{\lambda}\right)-\zeta\left(z+\frac{\mu}{\lambda} ; \frac{\tau_{1}}{\lambda}, \frac{\tau_{2}}{\lambda}\right)
$$

for some constant $\rho \in \mathbb{C}$. Here $\zeta(z)=\zeta\left(z ; \tau_{1}, \tau_{2}\right)$ is the Weierstrass zeta function

$$
\zeta\left(z ; \tau_{1}, \tau_{2}\right)=\frac{1}{z}+\sum_{\substack{\left.\omega=m_{1} \tau_{1}+m_{2} \tau_{2} \\\left(m_{1}, m_{2}\right) \in \mathbb{Z}^{2} \backslash(0,0)\right\}}}\left(\frac{1}{z-\omega}+\frac{1}{\omega}+\frac{z}{\omega^{2}}\right) .
$$

With the aid of the formulas $\zeta\left(\lambda z ; \lambda \tau_{1}, \lambda \tau_{2}\right)=\lambda^{-1} \zeta\left(z ; \tau_{1}, \tau_{2}\right)$ (see, for example, $\left[6\right.$ p.184]) and $\zeta(z)=\sigma^{\prime}(z) / \sigma(z)$,

$$
\begin{aligned}
\frac{\alpha^{\prime}(z)}{\alpha(z)} & =\rho+\lambda \zeta\left(\lambda z+\nu ; \tau_{1}, \tau_{2}\right)-\lambda \zeta\left(\lambda z+\mu ; \tau_{1}, \tau_{2}\right) \\
& =\frac{d}{d z} \log e^{\rho z} \frac{\sigma(\lambda z+\nu)}{\sigma(\lambda z+\mu)} .
\end{aligned}
$$

Thus

$$
\alpha(z)=C e^{\rho z} \frac{\sigma(\lambda z+\nu)}{\sigma(\lambda z+\mu)} .
$$

From $\alpha(0)=1, C=\sigma(\mu) / \sigma(\nu)$. Therefore we obtain

$$
\alpha(z)=e^{\rho z} \frac{\sigma(\mu) \sigma(\lambda z+\nu)}{\sigma(\nu) \sigma(\lambda z+\mu)} .
$$

This completes the proof of Theorem 4.3.

\section{Proposition 4.4.}

$$
\eta(z)=\lambda \zeta\left(\lambda z ; \tau_{1}, \tau_{2}\right)-\lambda \zeta\left(\lambda z+\mu ; \tau_{1}, \tau_{2}\right)+A,
$$


for some constant $A \in \mathbb{C}$.

Proof. By the theorem above

$\alpha(s)-\alpha(t) \alpha(s-t)$

$$
\begin{aligned}
= & e^{\rho s}\left(\frac{\sigma(\mu) \sigma(\lambda s+\nu)}{\sigma(\nu) \sigma(\lambda s+\mu)}-\frac{\sigma(\mu) \sigma(\lambda t+\nu) \sigma(\mu) \sigma(\lambda(s-t)+\nu)}{\sigma(\nu) \sigma(\lambda t+\mu) \sigma(\nu) \sigma(\lambda(s-t)+\mu)}\right) \\
= & e^{\rho s} \frac{\sigma(\mu)}{\sigma^{2}(\nu) \sigma(\lambda s+\mu) \sigma(\lambda t+\mu) \sigma(\lambda(s-t)+\mu)} \\
& \times\{\sigma(\nu) \sigma(\lambda t+\mu) \sigma(\lambda(s-t)+\mu) \sigma(\lambda s+\nu) \\
& -\sigma(\lambda s+\mu) \sigma(\mu) \sigma(\lambda t+\nu) \sigma(\lambda(s-t)+\nu)\} .
\end{aligned}
$$

We use the three term equation of $\sigma$ below.

$$
\begin{aligned}
& \sigma(x+y) \sigma(x-y) \sigma(z+w) \sigma(z-w) \\
+ & \sigma(x+z) \sigma(x-z) \sigma(w+y) \sigma(w-y) \\
+ & \sigma(x+w) \sigma(x-w) \sigma(y+z) \sigma(y-z) \\
= & 0 .
\end{aligned}
$$

By $\sigma(-x)=-\sigma(x)$,

$$
\alpha(s)-\alpha(t) \alpha(s-t)=e^{\rho s} \frac{\sigma(\mu) \sigma(\lambda s+\mu+\nu) \sigma(\mu-\nu) \sigma(\lambda(t-s)) \sigma(\lambda t)}{\sigma^{2}(\nu) \sigma(\lambda s+\mu) \sigma(\lambda t+\mu) \sigma(\lambda(s-t)+\mu)} .
$$

From the equation (1.4), we have

$$
\eta(t)=\lambda \zeta\left(\lambda t ; \tau_{1}, \tau_{2}\right)-\lambda \zeta\left(\lambda t+\mu ; \tau_{1}, \tau_{2}\right)+A,
$$

thereby completing the proof of Proposition 4.4.

\section{Lemma 4.5.}

$$
\varphi(z)=\exp (A z+B) \frac{\sigma(\lambda z)}{\sigma(\lambda z+\mu)},
$$

where $A$ is in Proposition 4.4, and $B \in \mathbb{C}$. 
Proof. $\quad$ By $\zeta(z)=(d / d z) \log \sigma(z)$,

$$
\begin{aligned}
\eta(z) & =A+\frac{d}{d z} \log \sigma(\lambda z)-\frac{d}{d z} \log \sigma(\lambda z+\mu) \\
& =\frac{d}{d z} \log \left(e^{A z} \frac{\sigma(\lambda z)}{\sigma(\lambda z+\mu)}\right) .
\end{aligned}
$$

From $\eta(z)=(d / d z) \log \varphi(z)$

$$
\log \varphi(z)=\log \left(e^{A z} \frac{\sigma(\lambda z)}{\sigma(\lambda z+\mu)}\right)+B
$$

Thus

$$
\varphi(z)=e^{A z+B} \frac{\sigma(\lambda z)}{\sigma(\lambda z+\mu)} .
$$

We have completed the proof of Lemma 4.5.

\section{Lemma 4.6.}

$$
\phi(z)=\exp ((\rho-A) z-2 B) \frac{\sigma(\mu) \sigma(\nu-\mu) \sigma(\lambda z+\mu+\nu)}{\sigma^{2}(\nu) \sigma(\lambda z+\mu)} .
$$

Proof. In the proof of Proposition 4.4, we obtain

$$
\alpha(s)-\alpha(t) \alpha(s-t)=e^{\rho s} \frac{\sigma(\mu) \sigma(\lambda s+\mu+\nu) \sigma(\mu-\nu) \sigma(\lambda(t-s)) \sigma(\lambda t)}{\sigma^{2}(\nu) \sigma(\lambda s+\mu) \sigma(\lambda t+\mu) \sigma(\lambda(s-t)+\mu)} .
$$

On the other hand,

$$
\varphi(t) \varphi(s-t)=e^{A s+2 B} \frac{\sigma(\lambda t) \rho(\lambda(s-t))}{\sigma(\lambda t+\mu) \sigma(\lambda(s-t)+\mu)} .
$$

From $\phi(s)=(\alpha(s)-\alpha(t) \alpha(s-t)) /(\varphi(t) \varphi(s-t))$, we get the desired result.

\section{§ 5. Degenerate Case, I}

Now we consider the most degenerate case $\Lambda_{+}=\{0\}$. The main result of this section is 
Theorem 5.1. If $\Lambda_{+}=\{0\}$, then

$$
\alpha(x)=e^{o x} \frac{\alpha x+b}{c x+b}
$$

for some $\rho, a, b$ and $c \in \mathbb{C}$ with $b(a-c) \neq 0$.

Conversely we deduce the following from some straightforward computation.

Proposition 5.2. The function $\alpha(x)=e^{o x}(\alpha x+b)(c x+b)^{-1}$ given in Theorem 5.1 satisfies the equation (0.2):

$$
\alpha(x) \alpha^{\prime}(y)-\alpha^{\prime}(x) \alpha(y)=(\alpha(x+y)-\alpha(x) \alpha(y))(\eta(x)-\eta(y)) .
$$

Here $\eta(x)$ is given by

$$
\eta(x)=\frac{b}{x(c x+b)}+A
$$

for an arbitrary constant $A \in \mathbb{C}$.

Because of $\eta(x)=\left(\varphi^{\prime}(x) / \varphi(x)\right)$, we have

$$
\varphi(x)=e^{A x+B} \frac{x}{c x+b}
$$

for an arbitrary constant $B \in \mathbb{C}$. Furthermore $\phi(x)$ is given by

$$
\phi(x)=e^{(\rho-A) x-2 B(c-a)\{a c x+b(a+c)\}} .
$$

Now we will prove Theorem 5.1.

Proof of Theorem 5.1. Choose a generic point $s \in \mathbb{C}$ such that $\alpha(s)$ $\neq \infty$ and $\psi(s) \neq 0, \infty$. Consider the meromorphic function of $t$

$$
h_{s}(t):=\alpha(t) \alpha(s-t)
$$

defined on the whole plane $\mathbb{C}$. From the equation (1.3)

$$
\frac{\alpha(s)-h_{s}(t)}{\varphi(t) \varphi(s-t)}=\phi(s) \neq 0, \infty,
$$

we have $h_{s}(t)=\alpha(s)$, if and only if $\varphi(t)=0$ or $\varphi(s-t)=0$. The latter condition is equivalent to $t=0$ or $s$ because of $\Lambda_{+}=\{0\}$. Moreover the function $h_{s}(t)=\alpha(t) \alpha(s-t)$ has at most two zeroes and at most two 
poles on $\mathbb{C}$ from Corollary 3.3 and Lemma 3.4 (2). Therefore the meromorphic function $h_{s}$ defined on the whole plane $\mathbb{C}$ has three exceptional values $0, \infty$ and $\alpha(s)$ for such a generic $s$. In view of the great Picard theorem (see, for example, [5]), the function $h_{s}$ extends itself to a meromorphic function defined on the whole Riemann sphere $\mathbb{C} \cup\{\infty\}$.

Recall $\Lambda^{\alpha} \leq 1$ and $\Lambda_{-} \leq 1$. In other words, we have $\Lambda^{\alpha}=\emptyset$ or $\left\{\nu_{0}\right\}$ and $\Lambda_{-}=\emptyset$ or $\left\{\mu_{0}\right\}$ for some $\nu_{0}$ and $\mu_{0} \in \mathbb{C}$. So we have four possibilities:

$$
\left(\# \Lambda^{\alpha}, \# \Lambda_{-}\right)=(0,0),(1,0),(0,1) \text { and }(1,1) \text {. }
$$

Introduce a linear fraction $S(x)$ by

$$
S(x):=1, x-\nu_{0}, \frac{1}{x-\mu_{0}} \text { and } \frac{x-\nu_{0}}{x-\mu_{0}},
$$

respectively. Then we have $\alpha(x) / S(x)=e^{g(x)}$ for some entire function $g$. In fact, Lemmas 3.2 and 3.4 imply $\operatorname{ord}_{\nu_{0}} \alpha=1$ and $\operatorname{ord}_{\mu_{0}} \alpha=-1$, respectively. For a generic $s, e^{g(t)+g(s-t)}=h_{s}(t) / S(t) S(s-t)$ is also a meromorphic function of $t$ on the whole $\mathbb{C} \cup\{\infty\}$, and furthermore it has no poles and no zeroes on $\mathbb{C}$. Therefore we have $e^{g(t)+g(s-t)}=c(s)$ for some constant $c(s)$ depending only on $s$. Differentiating it by the variable $t$, we obtain $g^{\prime}(t)-g^{\prime}(s-t)=0$ for any $t \in \mathbb{C}$ and a generic $s$.

Consequently the derivative $g^{\prime}$ is constant, so that $\alpha(x)=C e^{\rho x} S(x)$ for some $C$ and $\rho \in \mathbb{C}$. Since $\alpha$ is not obvious, we have $S \neq 1$. Recall $\alpha(0)$ $=1$ from Lemma 2.2. It follows that $\alpha(x)=e^{\rho x}(a x+b)(c x+b)^{-1}$ for some $a, b$ and $c \in \mathbb{C}$ with $b(a-c) \neq 0$. This completes the proof of Theorem 5.1.

\section{§ 6. Degenerate Case, II}

Finally we consider the singly-periodic case $\Lambda_{+} \cong \mathbb{Z}$.

Theorem 6.1. If $\Lambda_{+} \cong \mathbb{Z}$, then

$$
\alpha(x)=e^{o x} \frac{a\left(e^{2 x / \lambda}-1\right)+b}{c\left(e^{2 x / \lambda}-1\right)+b}
$$

for some $\rho, \lambda, a, b$ and $c \in \mathbb{C}$ with $\lambda \neq 0, b(a-c) \neq 0, a \neq 0$ or $b \neq c$, and $c \neq 0$ or $a \neq b$.

Proof. Fix a generator $\lambda_{0} \in \Lambda_{+}$. There exists some $\rho^{\prime} \in \mathbb{C}$ such that $e^{\rho^{\prime \prime} \lambda_{0}} \alpha\left(\lambda_{0}\right)=1$, since $\alpha\left(\lambda_{0}\right) \neq 0, \infty$. (See Lemmas 2.1 (1) and 3.1.) Remark 
that $e^{\rho^{\prime} x} \alpha(x)$ is also a solution of the equation (0.2). So we may replace $\alpha(x)$ with $e^{\rho^{\prime} x} \alpha(x)$. Then, from (3.1), $\alpha\left(\lambda^{\prime}\right)=1$ for all $\lambda^{\prime} \in \Lambda_{+}$, so that

$$
\alpha\left(x+\lambda^{\prime}\right)=\alpha(x)
$$

for any $x \in \mathbb{C}$ and any $\lambda^{\prime} \in \Lambda_{+}$.

Choose a generic point $s \in \mathbb{C}$ such that $\alpha(s) \neq \infty$ and $\phi(s) \neq 0, \infty$. Consider the meromorphic function of $t$

$$
h_{s}(t):=\alpha(t) \alpha(s-t)
$$

defined on the whole plane $\mathbb{C}$. From the equation (1.3)

$$
\frac{\alpha(s)-h_{s}(t)}{\varphi(t) \varphi(s-t)}=\phi(s) \neq 0, \infty,
$$

we have $h_{s}(t)=\alpha(s)$, if and only if $\varphi(t)=0$ or $\varphi(s-t)=0$. The latter condition is equivalent to $t \in \Lambda_{+}$or $t \in s+\Lambda_{+}$.

From (6.1) there exists a meromorphic function $k_{s}=k_{s}(\xi)$ defined on $\mathbb{C}^{\times}:=\mathbb{C} \backslash\{0\}$ such that

$$
h_{s}(x)=k_{s}\left(e^{2 \pi \sqrt{-1} x / \lambda_{0}}\right) .
$$

From what we have already shown, $k_{s}(\xi)=\alpha(s)$, if and only if $\xi=1$ or $e^{2 \pi \sqrt{-1} s / \lambda_{0}}$. Corollary 3.3 and Lemma 3.4 (2) imply that $k_{s}$ has at most two zeroes and at most two poles on $\mathbb{C}^{\times}$. Therefore the meromorphic function $k_{s}$ defined on $\mathbb{C}^{\times}$has three exceptional values $0, \infty$ and $\alpha(s)$ for such a generic $s$. In view of the great Picard theorem (see, for example, [5]), the function $k_{s}$ extends itself to a meromorphic function defined on the whole Riemann sphere $\mathbb{C} \cup\{\infty\}$.

Recall $\# \Lambda^{\alpha} / \Lambda_{+} \leq 1$ and $\# \Lambda_{-} / \Lambda_{+} \leq 1$. In other words, we have $\Lambda^{\alpha}=\emptyset$ or $\nu_{0}+\Lambda_{+}$and $\Lambda_{-}=\emptyset$ or $\mu_{0}+\Lambda_{+}$for some $\nu_{0}$ and $\mu_{0} \in \mathbb{C}$. So we have four possibilities:

$$
\left(\# \Lambda_{-} / \Lambda_{+}, \# \Lambda^{\alpha} / \Lambda_{+}\right)=(0,0),(0,1),(1,0) \text { and }(1,1) \text {. }
$$

Set $c^{\prime}:=e^{2 \pi \sqrt{-1} \nu_{0} / \lambda_{0}}$ and $c^{\prime \prime}:=e^{2 \pi \sqrt{-1} \mu_{0} / \lambda_{0}}$. We introduce a linear fraction $S(\xi)$ by

$$
S(\xi):=1, \xi-c^{\prime}, \frac{1}{\xi-c^{\prime \prime}}, \text { and } \frac{\xi-c^{\prime}}{\xi-c^{\prime \prime}},
$$

respectively. Then there exists some holomorphic function $g$ defined on the whole plane $\mathbb{C}$ such that 


$$
\alpha(x)=e^{g(x)} S\left(e^{2 \pi \sqrt{-1} x / \lambda_{0}}\right)
$$

from Lemmas 3.2 and 3.4 .

Set $\xi_{s}:=e^{2 \pi \sqrt{-1} s / \lambda_{0}}$ for $s \in \mathbb{C}$. Then, for a generic point $s$, the function $k_{s}(\xi) / S(\xi) S\left(\xi^{-1} \xi_{s}\right)$ is a meromorphic function defined on the whole $\mathbb{C} \cup\{\infty\}$, and has no poles and no zeroes on $\mathbb{C}^{\times}$. Therefore $k_{s}(\xi) / S(\xi) S\left(\xi^{-1} \xi_{s}\right)=b(s) \xi^{n(s)}$ for some function $b(s)$ and some integer $n(s) \in \mathbb{Z}$. From the definition of $k_{s}$,

$$
\begin{aligned}
e^{g(t)+g(s-t)} & =\alpha(t) \alpha(s-t) / S\left(e^{2 \pi \sqrt{-1} t / \lambda_{0}}\right) S\left(e^{2 \pi \sqrt{-1}(s-t) / \lambda_{0}}\right) \\
& =b(s) e^{2 \pi \sqrt{-1} n(s) t / \lambda_{0}} .
\end{aligned}
$$

Differentiating it by the variable $t$, we obtain

$$
g^{\prime}(t)-g^{\prime}(s-t)-2 \pi \sqrt{-1} n(s) / \lambda_{0}=0 .
$$

Differentiating it by the variable $t$ again, we get

$$
g^{\prime \prime}(t)+g^{\prime \prime}(s-t)=0
$$

for any $s$ and $t \in \mathbb{C}$. Hence $g^{\prime \prime}(t)=0$ and

$$
\alpha(x)=e^{g(x)} S\left(e^{2 \pi \sqrt{-1} x / \lambda_{0}}\right)=C e^{\rho x} S\left(e^{2 \pi \sqrt{-1} x / \lambda_{0}}\right)
$$

for some $C$ and $\rho \in \mathbb{C}$. Since $\alpha$ is not obvious, we have $S \neq 1$. Recall $\alpha(0)$ $=1$ from Lemma 2.2. Define $\lambda:=\lambda_{0} / \pi \sqrt{-1}$. Then we obtain

$$
\alpha(x)=e^{o x} \frac{\alpha\left(e^{2 x / \lambda}-1\right)+b}{c\left(e^{2 x / \lambda}-1\right)+b}
$$

for some $a, b$ and $c \in \mathbb{C}$ with $b(a-c) \neq 0$.

If $a=0$ and $b=c$, or if $c=0$ and $a=b$, then $\alpha$ is obvious. This completes the proof of Theorem 6.1.

By some straightforward computation, we obtain

\section{Lemma 6.2。}

$$
\alpha(x)=e^{o x} \frac{a\left(e^{2 x / \lambda}-1\right)+b}{c\left(e^{2 x / \lambda}-1\right)+b}
$$

satisfies the equation (0.2) 


$$
\alpha(x) \alpha^{\prime}(y)-\alpha^{\prime}(x) \alpha(y)=(\alpha(x+y)-\alpha(x) \alpha(y))(\eta(x)-\eta(y)),
$$

where

$$
\eta(x)=\frac{2 \lambda^{-1} e^{2 x / \lambda}}{e^{2 x / \lambda}-1}-\frac{2 \lambda^{-1} c e^{2 x / \lambda}}{c\left(e^{2 x / \lambda}-1\right)+b}+A
$$

for an arbitrary constant $A \in \mathbb{C}$.

\section{Lemma 6.3.}

$$
\varphi(x)=e^{A x+B} \frac{e^{2 x / \lambda}-1}{c\left(e^{2 x / \lambda}-1\right)+b},
$$

where the constant $A$ is in the lemma above and $B$ is an arbitrary constant.

Proof. It is trivial because of $\eta(x)=(d / d x) \log \varphi(x)$.

\section{Lemma 6.4.}

$$
\phi(x)=e^{-A x-2 B(a-c)\left\{-a c\left(e^{2 x / \lambda}-1\right)+b^{2}-b(a+c)\right\}} \frac{c\left(e^{2 x / \lambda}-1\right)+b}{.}
$$

Proof. From $\phi(s)=(\alpha(s)-\alpha(t) \alpha(s-t)) /(\varphi(t) \varphi(s-t))$, we get the desired result.

The solutions stated above are expressed in terms of the hyperbolic sine function.

Suppose $a \neq 0, b$ and $c \neq 0, b$. Then there exist $\mu$ and $\nu \in \mathbb{C}$ such that

$$
\left\{\begin{array}{l}
b=a\left(1-e^{-2 \nu}\right) \\
b=c\left(1-e^{-2 \mu}\right)
\end{array}\right.
$$

Then we get

$$
\begin{aligned}
\alpha(x) & =e^{\rho x} \frac{a\left(e^{2 x / \lambda}-1\right)+b}{c\left(e^{2 x / \lambda}-1\right)+b} \\
& =e^{\rho x} \frac{a\left(e^{2 x / \lambda}-1\right)+a\left(1-e^{-2 \nu}\right)}{c\left(e^{2 x / \lambda}-1\right)+c\left(1-e^{-2 \mu}\right)} \\
& =e^{\rho x} \frac{\sinh \mu \sinh \left(\lambda^{-1} x+\nu\right)}{\sinh \nu \sinh \left(\lambda^{-1} x+\mu\right)}
\end{aligned}
$$


These solutions are obtained in [1].

On the other hand, let $a=0$ and $c \neq b$, or let $a=b$ and $c \neq 0$. There exists $\mu \in \mathbb{C}$ such that $b=c\left(1-e^{-2 \mu}\right)$, and, as a result,

$$
\alpha(x)=e^{\left(\rho \mp \lambda^{-1}\right) x} \frac{\sinh \mu}{\sinh \left(\lambda^{-1} x+\mu\right)} .
$$

Next let $c=0$ and $a \neq b$, or let $b=c$ and $a \neq 0$. There exist $\nu \in \mathbb{C}$ such that $b=a\left(1-e^{-2 \nu}\right)$, and, as a result,

$$
\alpha(x)=e^{\left(p \pm \lambda^{-1}\right) x} \frac{\sinh \left(\lambda^{-1} x+\nu\right)}{\sinh \nu} .
$$

These two solutions are the limits of the solution (6.2) as $e^{ \pm 2 \nu}$ and $e^{ \pm 2 \mu}$ tend to 0 , respectively.

\section{References}

[1] Bruschi, M. and Calogero, F., The Lax representation for an integrable class of relativistic dynamical systems, Comm. Math. Phys., 109 (1987), 481-492.

[2] General analytic solution of certain functional equations of addition type, SIAM. J. Math. Anal., 21 (1990), 1019-1030.

[3] Calogero, F., Exactly solvable one-dimensional many-body problems, Lettere al Nuovo Cimento, 13 (1975), 411-416.

[4] On a functional equation connected with integrable many-body problems, Lettere al Nuovo Cimento, 16 (1976), 77-80.

[5] Conway, J. B., Functions of one complex variable, second edition, Springer-Verlag, New York, 1978.

[6] Hurwitz, A. and Courant, R., Vorlesungen über allgemeine Funktionentheorie und elliptische Funktionen, Springer-Verlag, Berlin, 1964.

[7] Moser, J., Three integrable Hamiltonian systems connected with isospectral deformations, Adv. Math., 16 (1975), 197-220.

[8] Ochiai, H., Oshima, T. and Sekiguchi, H., Commuting families of symmetric differential operators, Proc. Japan Acad., 70 (1994), 62-66.

[9] Olshanetsky, M. A. and Perelomov, A. M., Completely integrable Hamiltonian systems connected with semisimple Lie algebras, Invent. Math., 37 (1976), 93-108.

[10] Classical integrable finite-dimensional systems related to Lie algebras, Phys. Rep. (Review Section of Physics Letters), 71 (1981), 313-400.

[11] Integrable systems and Lie algebras, in Soviet Scientific Reviews, Section C, Math. Phys. Reviews 3 (S. P. Novikov, ed.), Harwood Academic Publishers GmbH, Switzerland, 1982, pp. 151-220.

[12] _ Integrable systems and finite-dimensional Lie algebras, in Dynamical systems VII (R. V. Gamkrelidze, ed.), Springer-Verlag, Berlin Heidelberg New York, 1994, pp. 87-116. 
[13] Oshima, T. and Sekiguchi, H., Commuting families of differential operators invariant under the action of a Weyl group, J. Math. Sci. Univ. Tokyo, 2 (1995), 175.

[14] Pontryagin, L. S., Topological Groups, 2nd edition, Gordon and Breach, New York, 1966.

[15] Ruijsenaars, S. N. M. and Schneider, H., A new class of integrable systems and its relation to solitons, Ann. Physics, 170 (1986), 370-405.

[16] Whittaker, E. T. and Watson, G. N., A course of modern analysis, 4th edition, Cambridge University Press, Cambridge, 1902. 
